\title{
Examining the Subacute Effects of Mild Traumatic Brain Injury Using a Traditional and Computerized Neuropsychological Test Battery
}

\author{
Rune Hatlestad Karlsen, ${ }^{1}$ Simen Berg Saksvik, ${ }^{2,3}$ Jonas Stenberg, ${ }^{1}$ Astri Johansen Lundervold, ${ }^{4}$ \\ Alexander Olsen, ${ }^{2,3}$ Ida Rautio, ${ }^{2}$ Line Folvik, ${ }^{2}$ Asta Kristine Håberg, ${ }^{1,5}$ Anne Vik, ${ }^{1,6}$ \\ Justin E. Karr, ${ }^{7}$ Grant L. Iverson, ${ }^{7}$ and Toril Skandsen ${ }^{1,3}$
}

\begin{abstract}
This study investigates subacute cognitive effects of mild traumatic brain injury (MTBI) in the Trondheim Mild TBI Study, as measured, in part, by the neuropsychological test battery of the Collaborative European NeuroTrauma Effectiveness Research in TBI (CENTER-TBI) program, including computerized tests from the Cambridge Neuropsychological Test Automated Battery (CANTAB) and traditional paper-and-pencil tests.

We investigated whether cognitive function was associated with injury severity: intracranial traumatic lesions on neuroimaging, witnessed loss of consciousness (LOC), or post-traumatic amnesia (PTA) $>1 \mathrm{~h}$. Further, we explored which of the tests in the CENTER-TBI battery might be associated with the largest subacute effects of MTBI (i.e., at 2 weeks postinjury). We recruited 177 patients with MTBI (16-59 years of age) from a regional trauma center and an outpatient clinic, 79 trauma control participants, and 81 community control participants. The MTBI group differed from community controls only on one traditional test of processing speed (coding; $p=0.009$, Cliff's delta $[\Delta]=0.20$ ). Patients with intracranial abnormalities performed worse than those without on a traditional test (phonemic verbal fluency; $p=0.043$, $\Delta=0.27$ ), and patients with LOC performed differently on the Attention Switching Task from the CANTAB ( $p=0.020$, $\Delta=-0.20)$. Patients with PTA $>1 \mathrm{~h}$ performed worse than those with $<1 \mathrm{~h}$ on 10 measures, from traditional tests and the CANTAB $(\Delta=0.33-0.20)$, likely attributable, at least in part, to pre-existing differences in intellectual functioning between groups. In general, those with MTBI had good neuropsychological outcome 2 weeks after injury and no particular CENTER-TBI computerized or traditional tests seemed to be more sensitive to subtle cognitive deficits.
\end{abstract}

Keywords: brain concussion; cognition; neuropsychology

\section{Introduction}

$\mathbf{T}$ HE MILD TRAUMATIC BRAIN INJURY (MTBI) classification is extremely broad, from injuries that are so mild they are difficult to detect ${ }^{1}$ to injuries that are complicated by skull fractures and macroscopic bleeding and contusions. ${ }^{2,3}$ It is well understood that there is enormous heterogeneity associated with injury mechanisms, ${ }^{4}$ pathophysiology, ${ }^{5}$ acute and subacute effects, ${ }^{6}$ and clinical recovery from MTBI. ${ }^{7,8}$ MTBI can have large adverse effects on cognitive functioning in the first few days following injury. ${ }^{8,9}$ Fortunately, performance on neuropsychological testing appears to normalize between 1 week and 3 months in the large majority of

\footnotetext{
${ }^{1}$ Department of Neuromedicine and Movement Science, Faculty of Medicine and Health Sciences, ${ }^{2}$ Department of Psychology, Faculty of Social and Educational Sciences, Norwegian University of Science and Technology, NTNU, Trondheim, Norway.

${ }^{3}$ Department of Physical Medicine and Rehabilitation, ${ }^{5}$ Department of Neurosurgery, ${ }^{6}$ Department of Neurosurgery, St. Olav's Hospital, Trondheim University Hospital, Trondheim, Norway.

${ }^{4}$ Department of Biological and Medical Psychology, University of Bergen, Bergen, Norway.

${ }^{7}$ Department of Physical Medicine and Rehabilitation, Harvard Medical School, Spaulding Rehabilitation Hospital and Spaulding Research Institute, Home Base Program, Red Sox Foundation and Massachusetts General Hospital, Charlestown, Massachusetts, USA.
}

(C) Rune Hatlestad Karlsen et al., 2020; Published by Mary Ann Liebert, Inc. This Open Access article is distributed under the terms of the Creative Commons Attribution Noncommercial License (CC-BY-NC) (http://creativecommons.org/licenses/by-nc/4.0/) which permits any noncommercial use, distribution, and reproduction in any medium, provided the original author(s) and the source are credited.

Correction added on March 1, 2021 after first online publication of October 30, 2020: The article reflects Open Access, with copyright transferring to the author(s), and a Creative Commons Attribution Noncommercial License (CC-BY-NC) added (http://creativecommons.org/ licenses/by-nc/4.0). 
people. $^{9-21}$ The nature and extent of cognitive deficits during the subacute period, from 2 to 3 weeks following injury, are not well understood because the majority of studies examine other timepoints, such as during the first week or at $1,3,6$, or 12 months following injury. Meta-analyses report small to medium effect sizes in the period 7 to 30 days post-injury, ${ }^{10,21}$ and findings from individual empirical studies on cognitive functioning in patients 2 to 3 weeks post-MTBI commonly report very small to medium effect sizes that often do not reach statistical significance, ${ }^{22-25}$ possibly due to insufficient statistical power.

Collaborative European NeuroTrauma Effectiveness Research in TBI (CENTER-TBI) is a large-scale, multi-national, European Commission-funded, longitudinal observational TBI research program. ${ }^{26-28}$ CENTER-TBI is generating repositories of comprehensive clinical patient data, neuroimaging, genetics, and biomarkers that can be used to try to improve diagnosis, stratification, management, and prognostication in brain injury medicine. ${ }^{27}$ The neuropsychological test battery used within CENTER-TBI includes a few traditional paper-and-pencil tests and a more comprehensive series of tests from the Cambridge Neuropsychological Test Automated Battery (CANTAB), a battery of computerized cognitive tests widely used in Europe in research and clinical trials. ${ }^{29-35}$ Previous meta-analyses have not identified meaningful differences in the cognitive effects of MTBI as detected by computerized tests versus paper-and-pencil tests, which produced similar effect sizes, ${ }^{13}$ although a trend for higher sensitivity of paper-and-pencil tests has also been reported. ${ }^{9}$

The purpose of this study was to examine the nature and extent of cognitive deficits at approximately 2 weeks following MTBI, using both traditional paper-and-pencil tests and tests from the CANTAB. Our selection of tests covered all the CENTER-TBI tests but included other tests as well. In addition, we explored which CENTER-TBI tests might be most sensitive to potential deficits. This is the first study, to our knowledge, to report effect sizes from the CENTER-TBI neuropsychological tests. This study could not be conducted using CENTER-TBI data because that longitudinal study did not include a control group.

The CANTAB has been developed to be sensitive and applicable in patients with cognitive impairments, ${ }^{36}$ but has rarely been used in MTBI research, and therefore, we sought to explore whether CANTAB tests might be associated with larger subacute effects of MTBI than traditional tests. We hypothesized that there would be small-to-medium differences on both computerized and traditional paper-and-pencil neuropsychological tests between participants with and without MTBIs. We did not have specific hypotheses relating to whether computerized testing would be more or less sensitive compared with traditional testing. We hypothesized that the subgroup of patients with MTBIs who had abnormalities on computed tomography (CT) or magnetic resonance imaging (MRI; i.e., those with complicated MTBIs), loss of consciousness (LOC) at time of injury, and post-traumatic amnesia (PTA) $<1 \mathrm{~h}$ would perform worse on neuropsychological testing than those who did not have these indicators of more severe injury.

\section{Methods}

\section{Participants}

The sample included patients $(n=177)$ taking part in the extended Trondheim MTBI follow-up study, who had been examined with neuropsychological tests at 2 weeks after injury. The Trondheim MTBI Study enrolled 378 patients with MTBI between April 1,2014 , and December $5,2015,{ }^{4}$ and around half were recruited to an extended follow-up study involving repeated neuropsychological testing, ${ }^{37}$ blood sampling, ${ }^{38}$ and brain MRI. ${ }^{39}$ The participants were consecutively recruited from two emergency departments (EDs): St. Olav's Hospital, a regional Level 1 trauma center at Trondheim University Hospital, Norway, and Trondheim Municipal Emergency Clinic, an outpatient clinic at the hospital run by general practitioners. The recruitment procedures and epidemiological characteristics of this cohort have been reported previously. ${ }^{4}$ Around half of the patients who presented to these EDs with MTBI were enrolled in the Trondheim MTBI follow-up study. There were only minor differences between enrolled and nonenrolled patients: enrolled patients were more often outpatients, women, injured during the weekend, and victims of violence. ${ }^{4}$ Our hospital was also a participating site in CENTER-TBI, and a subsample $(n=29)$ of the 177 patients in this study were also enrolled in CENTER-TBI.

Two comparison groups were recruited: 1) a community control sample (CC; $n=81$ ), matched for sex, age, and education, recruited among staff and students along with the social networks of staff, students, and patients; and 2) a trauma control sample (TC; $n=79$ ) with minor orthopedic injuries, matched for sex and age with the MTBI group, recruited from the same municipal EDs as the patients with MTBI. TCs were identified by screening of referrals to X-ray examination of the chest or extremities. The Central Regional Committee for Medical and Health Research Ethics approved the study (REK 2013/754). Participants, and parents of participants under 18 years old, gave informed consent. Participants were compensated with gift cards worth approximately \$US60 per visit. The study was performed in accordance with the Declaration of Helsinki.

\section{Inclusion and exclusion criteria}

TBI was categorized as mild according to the World Health Organization (WHO) Collaborating Center Task Force on Mild Traumatic Brain Injury criteria: Glasgow Coma Scale (GCS) score of $13-15$ at presentation to the ED; LOC, if present, $<30 \mathrm{~min}$; and PTA, if present, $<24 \mathrm{~h} .{ }^{40}$ Orthopedic injuries were musculoskeletal injuries not involving the head or neck, examined at the municipal ED with symptoms affecting normal function for at least 2 days.

Inclusion criteria for the MTBI group were; 1) having sustained MTBI; and 2) age 16.0-59.9 years. Exclusion criteria for all groups were: 1) non-residency in Norway or non-fluency in the Norwegian language; 2) ongoing severe somatic disease; 3) ongoing severe psychiatric disease or substance use problems, potentially making follow-up difficult; 4) pre-injury history of complicated mild, moderate, or severe TBI or other pre-existing neurological conditions with visible brain pathology or known cognitive deficits; 5) MTBI during the last 3 months; 6) presentation $>48 \mathrm{~h}$ after the initial trauma; and 7) other concurrent major trauma, such as spinal cord injury, severe fractures, or internal injuries. In addition, TCs were excluded if they had sustained an injury to their head, neck, or dominant upper extremity, and CCs were excluded if they were currently receiving psychiatric treatment.

\section{Demographic and injury-related variables}

Demographic variables were age, sex, and years of education. Injury-related variables such as cause of injury, GCS score, and level of care were defined and described in our previous publication. ${ }^{4}$ Most relevant in this study is LOC, which was categorized as "yes" if witnessed (i.e., if only self-reported, LOC was classified as no LOC), and duration of PTA, which was recorded as the time after injury for which the patient had no continuous memory. From a pilot study, we knew that many participants would not be able to provide a valid estimate in minutes, therefore PTA duration was reported as either $<1 \mathrm{~h}$ or $1-24 \mathrm{~h}$. 


\section{Neuroimaging}

Non-contrast head CT was performed on a Siemens Somatom Sensation 64-row scanner as part of the initial clinical assessment. CT findings were obtained from the radiology report and traumatic intracranial lesions (with or without additional fractures) were categorized as present or absent. Brain MRI was performed on a 3.0 Tesla Siemens Skyra system (Siemens Healthcare, Erlangen, Germany) with a 32-channel head coil for all patients at a mean $52 \mathrm{~h}$ (standard deviation $[\mathrm{SD}]=19 \mathrm{~h}$ ) after injury. The scan protocol consisted of: 1) three-dimensional (3D) T1-weighted magnetization prepared rapid gradient echo (MP-RAGE); (2) two-dimensional (2D) transversal diffusion weighted imaging; (3) 3D T2 space; 4) 3D fluid-attenuated inversion recovery (FLAIR); and 5) 3D suspectibility-weighted imaging (SWI). The images were first inspected by a resident in radiology, then reviewed by a dedicated, experienced neuroradiologist; and later, as part of a separate study, ${ }^{39}$ the images were reviewed again by a specialist in physical medicine and rehabilitation, and reported in consensus with the neuroradiologist. For the current study, MTBI was classified as uncomplicated if there were no intracranial traumatic lesions on either CT or MRI, and as complicated if any type or combination of intracranial lesions (i.e., contusion, traumatic axonal injury, or bleeding) was detected.

\section{Neuropsychological assessment}

All participants completed a comprehensive set of neuropsychological tests, including a selection of subtests from the CANTAB and paper-and-pencil tests, most of which are also included in the CENTER-TBI battery (Table 1). Patients with MTBI and TCs were administered the neuropsychological test battery approximately 2 weeks after their injury (mean \pm SD days since injury: $\mathrm{MTBI}=16.7 \pm 3.2$ days; $\mathrm{TC}=17.1 \pm 3.3$ days). Two weeks was chosen to reduce the acute general effects of trauma and medication, and to harmonize our data collection to that of CENTER-TBI. For CCs, the first neuropsychological assessment upon enrollment in the study was used. The tests were administrated by research staff with bachelor's or master's degrees in clinical psychology or neuroscience who were supervised by a licensed clinical psychologist (R.H.K).

\section{Paper-and-pencil tests}

Nine paper-and pencil neuropsychological tests (11 outcome measures) were included as part of the assessment procedure, and we analyzed raw scores as well as scores standardized according to published norms (Table 1). Administrative and psychometric properties of these tests have been reported in detail elsewhere. ${ }^{41,42}$ The Trail Making Test (TMT) provides information on visual attentional abilities, processing speed, and cognitive flexibility. ${ }^{41,42}$ The TMT consists of two parts. In TMT Part A, participants are instructed to draw a line connecting the numbers 1 to 25 as fast as possible. TMT Part B requires participants to draw a line alternating between the number (i.e., 1 to 13 ) and letter (i.e., A to L) sets. Time-to-completion is the primary outcome for each part of the TMT, and published norms were used to calculate age-adjusted T-scores for these outcomes. ${ }^{43}$

The Symbol Search test and the Coding test are both measures of processing speed from the Wechsler Adult Intelligence Scale, 4th edition (WAIS-IV). ${ }^{44,45}$ On the Symbol Search test, participants are required to decide if one of two target symbols is present in a sequence of other symbols. On the Coding test, participants are told to draw different symbols according to a digit-to-symbol key. The number of completed items serves as the outcome for both tests and norms from the Norwegian manual were used to calculate ageadjusted T-scores. $^{44}$

Table 1. Tests Included in the Trondheim MTBI Follow-Up Study and in the CENTER-TBI

Trondheim Study test battery

AST Congruency Cost

AST Switch Cost

AST \% Correct

PAL Total Errors

PRM Immediate

PRM Delayed

RVP A prime

SRM \% Correct

SSP Span Length

SWM Between Errors

SWM Strategy

RTI Simple RT

RTI 5-choice RT

SOC Minimum Moves

Matrix Reasoning

Vocabulary

TMT Part A

TMT Part B

Verbal fluency - Words

Verbal fluency - Animals

AVLT 1 to 5

AVLT Immediate recall

AVLT Delayed recall

Symbol Search

Coding
Normative sample

CENTER-TBI battery

No norms

No norms

Norms provided with CANTAB

No norms

No norms

Norms provided with CANTAB

No norms

Norms provided with CANTAB

Norms provided with CANTAB

Norms provided with CANTAB

Norms provided with CANTAB

Norms provided with CANTAB

Norms provided with CANTAB

Wechsler, $1999^{48} ; 2007^{49}$

Wechsler, $1999^{48} ; 2007^{49}$

Mitrushina, $2005^{43}$

Mitrushina, $2005^{43}$

Tombaugh, $1999^{47}$

Tombaugh, $1999^{47}$

Schmidt, 1996, in Strauss, $2006^{41,46}$

Schmidt, 1996, in Strauss, $2006^{41,46}$

Schmidt, 1996, in Strauss, $2006^{41,46}$

Wechsler, 2008 ${ }^{44} ; 2011^{45}$

Wechsler, $2008^{44} ; 2011^{45}$
Yes

Yes

Yes

Yes

Yes

-

Yes

Yes

No

Yes

Yes

Yes

Yes

-

Yes

Yes

Yes

-

AST, Attention Switching Task; AVLT, Auditory Verbal Learning Test; CENTER-TBI, Collaborative European NeuroTrauma Effectiveness Research in TBI; MTBI, mild TBI; PAL, Paired Associated Learning; PRM, Pattern Recognition Memory; RTI, Reaction Time; RVP, Rapid Visual Processing; SOC, Stockings of Cambridge; SRM, Spatial Recognition Memory; SSP, Spatial Span; SWM, Spatial Working Memory; TBI, traumatic brain injury; TMT, Trail Making Test. 
The Rey Auditory Verbal Learning Test (RAVLT) is a test of verbal learning and memory. ${ }^{41} \mathrm{~A}$ list of 15 words is read aloud five times and participants are asked to recall as many words as possible after each trial. A distractor list is provided and participants are asked to recall the original list immediately after the distractor list and again after a 20-min delay. The sum of words recalled on trials $1-5$, the immediate recall of the list after being presented a distracter list, and the delayed recall were used as outcomes. Published norms were used to calculate age-adjusted T-scores. ${ }^{41,46}$ Verbal fluency is a test of language and executive functioning. ${ }^{41}$ In the phonemic trial, participants are asked to generate as many words as possible beginning with a specific letter within $60 \mathrm{sec}$, including three trials with three different letters. On the semantic fluency trial, participants are asked to name as many animals as possible in $60 \mathrm{sec}$. The number of words produced within the time limit serves as the primary outcome for both fluency tests, for which published norms were used to calculate age-adjusted T-scores. ${ }^{47}$

Two tests from the Wechsler Abbreviated Scale of Intelligence (WASI) were used to assess general intellectual functioning. ${ }^{48,49}$ On the Vocabulary test, a measure of verbal comprehension, participants are told to define different words. On the Matrix Reasoning task, a measure of visual reasoning skills, participants are instructed to identify patterns among complex visual images. The number of correct responses is the primary outcome for each WASI test, with norms from the WASI manual being used to calculate age-adjusted T-scores. ${ }^{48,49}$

\section{CANTAB}

Fourteen outcome measures from nine CANTAB tests were included in the assessment procedure. Three tests were assumed to measure visual learning and memory. The Paired Associates Learning (PAL) task presents participants with several white boxes that contain different patterns. Each pattern is subsequently revealed for $1 \mathrm{sec}$ and the participants must remember which box contains which pattern. Total errors adjusted for the number of trials was chosen as the outcome measure. The Pattern Recognition Memory (PRM) test presents participants with two different series of 12 patterns. Participants are then required to identify previously seen patterns among novel patterns immediately after presentation (the first series) and after a 20-min delay (second series). The percent of correctly identified patterns for each trial was chosen as the outcome measure. The Spatial Recognition Memory (SRM) test presents the participants with a sequence of five white boxes appearing at various positions on the screen. Participants must remember the screen placement for each of the boxes. The percent correct was chosen as the outcome measure for the SRM.

Four tests were assumed to measure executive function. In the Stockings of Cambridge (SOC) test, participants are shown two displays with three balls presented inside stockings, and the aim is to move the balls in the lower display such that it is identical to the arrangement of balls in the upper display. Problems solved with the minimum possible moves was chosen as the outcome measure.

In the Attention Switching Task (AST), participants are to determine the side or direction of an arrow on the screen. The arrow varies with respect to placement (right or left) and direction (right or left). Three outcome measures were chosen. The first outcome measure, congruency cost, is the difference in mean response time in milliseconds on congruent (placement and direction are the same) and incongruent (placement and direction are not the same) trials. A positive score indicates that the participant is faster on congruent trials and a negative score indicates that the participant is faster on incongruent trials. The second outcome measure, switch cost, is the difference in mean response time in milliseconds on non-switched trials (where the trial type and the previous trial type are the same, i.e., direction-direction or side-side) versus switched trials. A positive score indicates that the participant is faster on nonswitched trials, and a negative score indicates that the participant is faster on switched trials. For both congruency cost and switch cost we interpreted faster performance on incongruent/switched trials to be indicative of higher executive function ability, meaning a lower score was suggestive of better performance. The third outcome measure is the percent of correct trials.

The Spatial Working Memory (SWM) test requires participants to search through boxes for a designated number of tokens. A token is never hidden in the same box twice; and to avoid errors, participants must remember where tokens originally appeared. Two outcome measures were chosen. The first outcome measure, between errors, is defined as the number of times the participant revisits a box in which a token has previously been found. The second outcome measure quantifies the effectiveness of the participant's strategy. This is a measure of the ability to follow a predetermined sequence beginning with a specific box and then to return to that box to start a new sequence once a token has been found. The minimum strategy score is 8 and the maximum is 56. A higher score implies non-compliance to this strategy and indicates poor strategy use. The Spatial Span (SSP) test presents participants with multiple white boxes that change color one by one. Participants are asked to tap the boxes in the same order as they changed their color and maximum span length (longest sequence) was chosen as the outcome measure.

Two tests were assumed to measure visual attention. In the Rapid Visual Processing (RVP) test participants are presented with numbers from 2 to 9 appearing inside a white box one at a time with a rate of 100 presentations per minute. The participants must press a button on a response box each time they see one of three target sequences (three digits). A prime $\left(A^{\prime}\right)$ is a measure of the ability to detect the target sequence and is the relationship between the probability of identifying a target sequence and the probability of identifying a non-target sequence. It ranges from 0.00 to 1.00 and a higher score is indicative of better performance. In the Reaction Time (RTI) test, the participant responds as fast as possible when a yellow dot is presented inside a circle (simple reaction time) and in one of five white circles (five-choice reaction time). Response time in milliseconds for each condition (i.e., simple and five-choice) was chosen as the outcome measure, with a quicker response time indicative of better performance.

\section{Statistical analysis}

Outcome measures were screened for univariate outliers and transformed using winsorization with a trim value $\mathrm{Z} \pm 3.0$ in IBM SPSS Statistics. ${ }^{50}$ A total of 41 values were transformed (27 from MTBI, 5 from TC, and 9 from HC). Subsequent statistical analyses were conducted in $\mathrm{R}^{51}$ By investigating skew and kurtosis values, as well as conducting a Shapiro-Wilks test of normality, the large majority of outcome measures showed a non-normal distribution of scores. Visual inspection of density plots did not suggest differences between MTBI, CCs, and TCs. Differences between groups in the proportion of men and women were analyzed with a chi-square test and Kruskal-Wallis rank sum tests were used to analyze differences in age, education, and outcome measures between MTBI, CCs, and TCs. Post hoc tests of pairwise differences were analyzed with MannWhitney U tests. Differences in outcome measures between patients with and without complicated MTBI, LOC, and PTA $\geq 1 \mathrm{~h}$ were analyzed with Mann-Whitney U tests. We calculated effect size estimates with Cliff's delta $(\Delta)$, coded such that worse cognitive outcome in the presumed most affected group would result in a positive effect size. The Cliff's delta is a measure of overlap between two distributions. An effect size of 1.0 or -1.0 indicates no overlap, whereas an effect size of 0 indicates a complete overlap. A Cliff's delta of 0.11 is considered a small effect size, 0.28 is considered a medium effect size, and 0.43 a large effect size. ${ }^{52}$ Notably, no corrections for multiple comparisons were applied because this study was designed to comprehensively explore a large number of individual neuropsychological tests within the CENTER-TBI battery, with an emphasis on effect size interpretation as opposed to significance testing. 


\section{Results}

\section{Representativeness of patients} with neuropsychological data

This study included the 177 patients with MTBI and neuropsychological test data. The other 201 patients with MTBI, not included in the extended study, had slightly fewer years of education. Otherwise, no significant differences were found in patient characteristics based on the availability of neuropsychological test data (Table 2).

\section{Characteristics of the MTBI group and the control groups}

There were no differences in age, sex, or education between patients with MTBI, TCs, and CCs, but the MTBI group had a higher frequency of previous MTBI than both control groups (Table 2). LOC was observed in 92 patients $(47 \%)$ and PTA was $\geq 1 \mathrm{~h}$ in 52 patients (29\%). MRI brain scans were available for all but four MTBI patients. Two of those without an MRI scan had intracranial lesions on the day-of-injury CT and one had a normal CT. In one case, no neuroimaging was performed, and this case was therefore removed from the subgroup analyses on complicated MTBI. Hence, 21 patients $(12 \%)$ were included in the complicated MTBI group.

\section{Group comparisons in neuropsychological test performances}

The Coding subtest was the only test with a statistically significant group difference $(p=0.025)$, where post hoc analyses showed significantly worse performance in the MTBI group compared with the CC group ( $p=0.009, \Delta=0.20$ ), but not compared with the TC group ( $p=0.216, \Delta=0.10$; Table 3). Comparing the three groups, no statistically significant differences were found for any other tests, and the Cliff's delta effect sizes were uniformly small to very small. The MTBI group and the control groups all performed in the average range according to published norms. When we compared the proportion of participants in the different groups performing below a cutoff at 1.5 SD according to the norms, no significant differences were observed (Table 4).

\section{Comparisons in neuropsychological test performances by injury severity indices}

There were no statistically significant differences in age, sex, or years of education between the complicated $(n=21)$ and uncomplicated groups $(n=156)$. A statistically significant difference in test performance was observed for the Verbal fluency test (phonemic trial), with worse performance in the complicated group

Table 2. Demographic and Clinical Characteristics of Patients, Trauma Controls, and Community Controls

\begin{tabular}{|c|c|c|c|c|}
\hline Variable & $\begin{array}{c}\text { Participants } \\
\text { with } M T B I \\
\text { No NP }(\mathrm{n}=201)\end{array}$ & $\begin{array}{c}\text { Participants } \\
\text { with MTBI } \\
N P(\mathrm{n}=177)\end{array}$ & $\begin{array}{l}\text { Trauma control } \\
\text { participants } \\
(\mathrm{n}=79)\end{array}$ & $\begin{array}{l}\text { Community control } \\
\text { participants } \\
(\mathrm{n}=81)\end{array}$ \\
\hline Age, median (IQR) & $24(20-37)$ & $28(22-45)$ & $29(22-46)$ & $28(23-44)$ \\
\hline Age, mean (SD) & $29.7(12.6)$ & $33.0(13.3)$ & $32.8(13.2)$ & $33.2(13.1)$ \\
\hline Men, $n(\%)$ & $134(67)$ & $113(64)$ & $49(62)$ & $50(62)$ \\
\hline Years of education ${ }^{a}$ & $13(13-15)$ & $13(13-16)^{*, \#}$ & $14.4(2.6)$ & $14.0(2.4)$ \\
\hline Previous MTBI, yes, $n(\%)$ & $39(20)^{\mathrm{b}}$ & 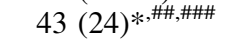 & $5(7)^{c}$ & $10(12)$ \\
\hline \multicolumn{5}{|l|}{ Cause of injury, $n(\%)$} \\
\hline Fall & $68(35)$ & $67(38)$ & $24(30)$ & - \\
\hline Violence & $42(21)$ & $23(13)$ & $1(1)$ & - \\
\hline Bicycle & $24(12)$ & 34 (19) & $7(9)$ & - \\
\hline Sport & $31(16)$ & $23(13)$ & $29(37)$ & - \\
\hline MVA & $27(14)$ & $16(9)$ & $3(4)$ & - \\
\hline Struck object & $5(3)$ & $12(7)$ & $6(8)$ & - \\
\hline Other & $2(1)$ & $1(1)$ & $9(11)$ & - \\
\hline Unknown & $2(1)$ & $1(1)$ & - & - \\
\hline \multicolumn{5}{|l|}{ GCS score, $n(\%)$} \\
\hline 15 & $138(69)^{\mathrm{d}}$ & $149(77)^{\mathrm{e}}$ & - & - \\
\hline 14 & 34 (17) & $30(16)$ & - & - \\
\hline 13 & $1(1)$ & $4(2)$ & - & - \\
\hline Missing & $28(14)$ & $11(6)$ & - & - \\
\hline Witnessed LOC, $n(\%)$ & $86(43)$ & 87 (49) & - & - \\
\hline PTA $>1$ & $55(27)$ & $52(29)$ & - & - \\
\hline Intracranial traumatic CT findings & $11(7)$ & $11(8)$ & - & - \\
\hline Complicated MTBI (incl MRI) & MRI not performed & $21(12)$ & - & - \\
\hline \multicolumn{5}{|l|}{ Level of care, $n(\%)$} \\
\hline Discharged home from ED & $136(68)$ & $124(70)$ & $68(86)$ & - \\
\hline Observation $<24 \mathrm{~h}$ & $34(17)$ & $27(15)$ & - & - \\
\hline Neurosurgical admission & $22(11)$ & $17(10)$ & - & - \\
\hline Orthopedic/other admission & $9(5)$ & $9(5)$ & $11(4)$ & - \\
\hline
\end{tabular}

* $p<0.05$; \#comparison between No NP and NP; \#\#comparison between NP and trauma controls; \#\#\#comparison between NP and community controls, a: $n=198$, b: $n=197$, c: $n=76$, d: $n=173$, e: $n=166$.

CT, computed tomography; ED, emergency department; GCS, Glasgow Coma Scale; IQR, interquartile range; LOC, loss of consciousness; MRI, magnetic resonance imaging; MTBI, mild traumatic brain injury; MVA, motor vehicle accident; NP, neuropsychological tests performed; PTA, posttraumatic amnesia; SD, standard deviation. 


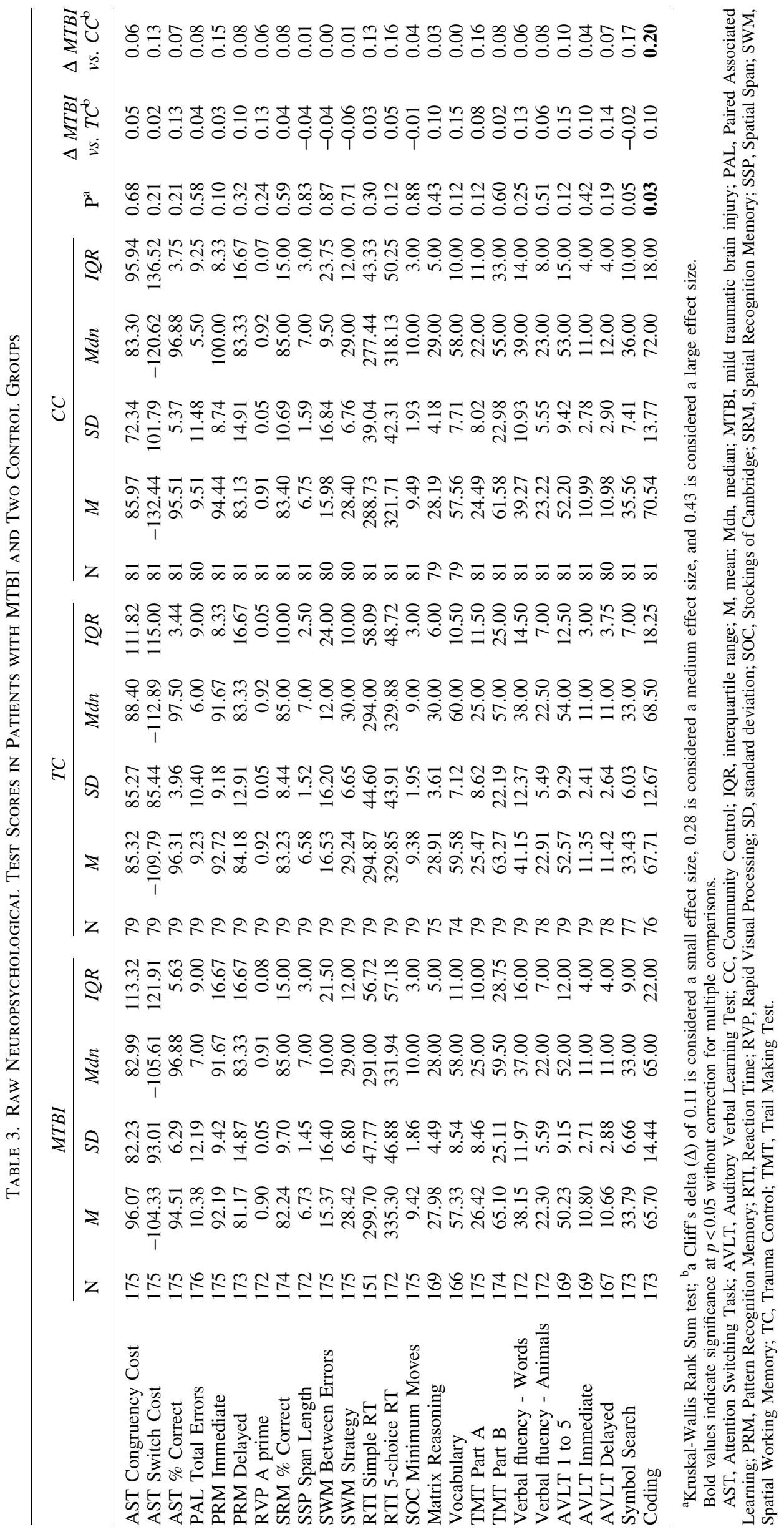


Table 4. T-Scores and Proportion of Patients and Control Subjects Scoring Below 1.5 SD

\begin{tabular}{|c|c|c|c|c|c|c|c|c|c|c|c|c|c|}
\hline & \multicolumn{4}{|c|}{ MTBI } & \multicolumn{4}{|c|}{$T C$} & \multicolumn{4}{|c|}{$C C$} & \multirow[b]{2}{*}{$\mathrm{P}^{\mathrm{a}}$} \\
\hline & $\mathrm{N}$ & $M$ & $S D$ & $\%$ Abnormal & $\mathrm{N}$ & $M$ & $S D$ & $\%$ Abnormal & $\mathrm{N}$ & $M$ & $S D$ & $\%$ Abnormal & \\
\hline PAL Total Errors & 167 & 50.82 & 7.44 & 2.40 & 77 & 51.34 & 5.96 & 1.30 & 78 & 51.94 & 6.38 & 2.56 & 0.83 \\
\hline RVP A prime & 172 & 46.05 & 12.65 & 19.19 & 79 & 49.22 & 10.44 & 10.13 & 81 & 47.70 & 10.68 & 13.58 & 0.16 \\
\hline SSP Span Length & 172 & 52.07 & 10.00 & 6.40 & 79 & 50.93 & 10.84 & 11.39 & 81 & 52.69 & 10.12 & 2.47 & 0.08 \\
\hline SWM Between Errors & 175 & 52.26 & 10.10 & 5.71 & 79 & 51.48 & 9.28 & 3.80 & 80 & 53.00 & 7.83 & 3.75 & 0.71 \\
\hline SWM Strategy & 175 & 54.42 & 12.04 & 5.14 & 79 & 52.99 & 11.89 & 5.06 & 80 & 54.71 & 10.83 & 0.00 & 0.12 \\
\hline RTI Simple Reaction Time & 151 & 52.92 & 10.35 & 5.96 & 79 & 53.94 & 9.03 & 2.53 & 81 & 55.18 & 8.36 & 2.47 & 0.31 \\
\hline RTI 5-choice Reaction Time & 172 & 51.81 & 11.04 & 5.23 & 79 & 53.38 & 8.71 & 5.06 & 81 & 55.22 & 8.02 & 0.00 & 0.11 \\
\hline SOC Minimum Moves & 175 & 51.42 & 9.89 & 9.10 & 79 & 51.25 & 10.05 & 6.30 & 81 & 52.14 & 9.88 & 7.40 & 0.73 \\
\hline Matrix Reasoning & 169 & 57.41 & 8.32 & 2.37 & 75 & 58.95 & 7.16 & 0.00 & 79 & 57.76 & 6.68 & 0.00 & 0.16 \\
\hline Vocabulary & 166 & 50.92 & 9.05 & 4.82 & 74 & 53.45 & 7.29 & 1.35 & 79 & 51.08 & 8.23 & 2.53 & 0.35 \\
\hline TMT A & 175 & 49.40 & 11.77 & 8.00 & 79 & 50.51 & 11.44 & 5.06 & 81 & 52.34 & 8.49 & 3.70 & 0.37 \\
\hline TMT B & 174 & 47.36 & 13.73 & 15.52 & 79 & 48.28 & 12.27 & 7.59 & 81 & 49.69 & 9.79 & 11.11 & 0.19 \\
\hline Verbal fluency - Words & 172 & 46.58 & 11.66 & 13.95 & 79 & 49.93 & 11.86 & 8.86 & 81 & 47.82 & 10.48 & 8.64 & 0.33 \\
\hline Verbal fluency - Animals & 172 & 53.70 & 11.64 & 5.23 & 78 & 54.69 & 11.65 & 3.85 & 81 & 55.83 & 12.12 & 1.23 & 0.31 \\
\hline AVLT 1 to 5 & 169 & 45.69 & 11.83 & 17.75 & 79 & 48.83 & 11.29 & 11.39 & 81 & 48.42 & 11.71 & 13.58 & 0.39 \\
\hline AVLT Immediate & 169 & 49.17 & 10.61 & 12.43 & 79 & 51.49 & 8.76 & 3.80 & 81 & 50.02 & 10.60 & 7.41 & 0.07 \\
\hline AVLT Delayed & 167 & 49.01 & 10.64 & 10.78 & 78 & 51.77 & 9.57 & 6.41 & 80 & 50.29 & 10.62 & 10.00 & 0.55 \\
\hline Symbol Search & 173 & 52.20 & 8.52 & 0.58 & 77 & 51.69 & 7.90 & 1.30 & 81 & 54.53 & 8.62 & 1.23 & 0.80 \\
\hline Coding & 173 & 50.39 & 8.83 & 1.16 & 76 & 51.67 & 7.75 & 0.00 & 81 & 53.29 & 8.14 & 0.00 & 0.40 \\
\hline
\end{tabular}

${ }^{\mathrm{a}}$ Chi-square test.

AVLT, Auditory Verbal Learning Test; CC, Community Control; M, mean; MTBI, mild traumatic brain injury; PAL, Paired Associated Learning; RTI, Reaction Time; RVP, Rapid Visual Processing; SD, standard deviation; SOC, Stockings of Cambridge; SSP, Spatial Span; SWM, Spatial Working Memory; TC, Trauma Control; TMT, Trail Making Test.

Table 5. Comparing Patients with Complicated and Uncomplicated MTBIs

\begin{tabular}{|c|c|c|c|c|c|c|c|c|c|c|c|c|}
\hline & \multicolumn{5}{|c|}{ Uncomplicated MTBI } & \multicolumn{5}{|c|}{ Complicated MTBI } & \multirow[b]{2}{*}{$\mathrm{P}^{\mathrm{a}}$} & \multirow[b]{2}{*}{$\Delta^{\mathrm{b}}$} \\
\hline & $\mathrm{N}$ & $M$ & $S D$ & $M d n$ & $I Q R$ & $\mathrm{~N}$ & $M$ & $S D$ & $M d n$ & $I Q R$ & & \\
\hline Men & $98(63 \%)$ & & & & & $15(71 \%)$ & & & & & 0.60 & \\
\hline Age & 156 & 33.31 & 13.47 & 29.24 & 24.01 & 21 & 30.31 & 11.52 & 24.66 & 12.19 & 0.60 & \\
\hline Education & 156 & 13.95 & 2.63 & 13.00 & 4.00 & 21 & 13.81 & 2.44 & 13.00 & 4.00 & 0.60 & \\
\hline AST Congruency Cost & 154 & 99.63 & 81.47 & 89.59 & 116.56 & 21 & 69.99 & 85.04 & 43.33 & 110.87 & 0.07 & -0.24 \\
\hline AST Switch Cost & 154 & -101.21 & 92.23 & -101.48 & 123.01 & 21 & -127.19 & 97.85 & -131.35 & 132.64 & 0.24 & -0.16 \\
\hline AST \% Correct & 154 & 94.27 & 6.54 & 96.25 & 6.25 & 21 & 96.31 & 3.58 & 96.88 & 2.50 & 0.32 & -0.13 \\
\hline PAL Total Errors & 155 & 10.60 & 12.32 & 7.00 & 9.00 & 21 & 8.76 & 11.35 & 6.00 & 5.00 & 0.51 & -0.09 \\
\hline PRM Immediate & 154 & 92.05 & 9.73 & 91.67 & 16.67 & 21 & 93.25 & 6.78 & 91.67 & 8.33 & 0.97 & 0.00 \\
\hline PRM Delayed & 152 & 81.36 & 14.57 & 83.33 & 16.67 & 21 & 79.76 & 17.19 & 83.33 & 16.67 & 0.91 & 0.02 \\
\hline RVP A prime & 151 & 0.90 & 0.05 & 0.90 & 0.07 & 21 & 0.92 & 0.06 & 0.92 & 0.10 & 0.20 & -0.17 \\
\hline SRM \% Correct & 153 & 82.58 & 9.58 & 85.00 & 15.00 & 21 & 79.76 & 10.43 & 80.00 & 10.00 & 0.19 & 0.17 \\
\hline SSP Span Length & 151 & 6.74 & 1.50 & 7.00 & 3.00 & 21 & 6.71 & 1.10 & 7.00 & 2.00 & 0.92 & 0.01 \\
\hline SWM Between Errors & 154 & 15.01 & 16.10 & 10.00 & 21.75 & 21 & 17.95 & 18.63 & 11.00 & 20.00 & 0.47 & 0.10 \\
\hline SWM Strategy & 154 & 28.25 & 6.80 & 28.50 & 12.00 & 21 & 29.67 & 6.84 & 30.00 & 9.00 & 0.42 & 0.11 \\
\hline RTI Simple Reaction Time & 134 & 298.20 & 45.96 & 290.17 & 54.44 & 17 & 311.52 & 60.56 & 293.89 & 74.78 & 0.60 & 0.08 \\
\hline RTI 5-choice Reaction Time & 151 & 333.71 & 45.46 & 330.13 & 54.50 & 21 & 346.72 & 55.96 & 342.50 & 72.63 & 0.33 & 0.13 \\
\hline SOC Minimum Moves & 154 & 9.36 & 1.90 & 9.00 & 3.00 & 21 & 9.86 & 1.53 & 10.00 & 2.00 & 0.25 & -0.15 \\
\hline Matrix Reasoning & 150 & 27.89 & 4.59 & 28.00 & 5.00 & 19 & 28.74 & 3.57 & 29.00 & 4.50 & 0.50 & -0.09 \\
\hline Vocabulary & 147 & 57.29 & 8.48 & 58.00 & 11.00 & 19 & 57.58 & 9.16 & 56.00 & 14.00 & 0.88 & -0.02 \\
\hline TMT A & 155 & 26.38 & 8.40 & 25.00 & 10.00 & 20 & 26.75 & 9.15 & 27.00 & 8.25 & 0.86 & 0.03 \\
\hline TMT B & 154 & 65.24 & 24.09 & 60.00 & 29.25 & 20 & 64.05 & 32.65 & 54.00 & 26.50 & 0.32 & -0.14 \\
\hline Verbal fluency - Words & 151 & 38.75 & 11.38 & 38.00 & 15.50 & 21 & 33.76 & 15.18 & 33.00 & 12.00 & 0.04 & 0.27 \\
\hline Verbal fluency - Animals & 151 & 22.37 & 5.41 & 23.00 & 7.00 & 21 & 21.76 & 6.89 & 20.00 & 9.00 & 0.29 & 0.14 \\
\hline AVLT 1 to 5 & 149 & 50.41 & 8.97 & 52.00 & 11.00 & 20 & 48.90 & 10.56 & 47.00 & 17.50 & 0.57 & 0.08 \\
\hline AVLT Immediate & 149 & 10.82 & 2.75 & 11.00 & 4.00 & 20 & 10.65 & 2.50 & 10.00 & 3.25 & 0.66 & 0.06 \\
\hline AVLT Delayed & 148 & 10.70 & 2.91 & 11.00 & 4.00 & 19 & 10.37 & 2.67 & 11.00 & 3.50 & 0.57 & 0.08 \\
\hline Symbol Search & 153 & 33.71 & 6.45 & 33.00 & 9.00 & 20 & 34.40 & 8.29 & 36.00 & 11.50 & 0.52 & -0.09 \\
\hline Coding & 153 & 66.16 & 14.54 & 65.00 & 22.00 & 20 & 62.15 & 13.44 & 64.00 & 15.75 & 0.33 & 0.14 \\
\hline
\end{tabular}

${ }^{\mathrm{a}}$ Mann-Whitney U test; ${ }^{\mathrm{b}}$ a Cliff's delta $(\Delta)$ of 0.11 is considered a small effect size, 0.28 is considered a medium effect size, and 0.43 is considered a large effect size.

Bold values indicate significance at $p<0.05$ without correction for multiple comparisons.

AST, Attention Switching Task; AVLT, Auditory Verbal Learning Test; IQR, interquartile range; M, mean; Mdn, median; MTBI, mild traumatic brain injury; PAL, Paired Associated Learning; PRM, Pattern Recognition Memory; RTI, Reaction Time; RVP, Rapid Visual Processing; SD, standard deviation; SOC, Stockings of Cambridge; SRM, Spatial Recognition Memory; SSP, Spatial Span; SWM, Spatial Working Memory; TMT, Trail Making Test. 
Table 6. Comparing Patients with and without LOC

\begin{tabular}{|c|c|c|c|c|c|c|c|c|c|c|c|c|}
\hline & \multicolumn{5}{|c|}{ No LOC } & \multicolumn{5}{|c|}{$L O C$} & \multirow[b]{2}{*}{$\mathrm{P}^{\mathrm{a}}$} & \multirow[b]{2}{*}{$\Delta^{\mathrm{b}}$} \\
\hline & $\mathrm{N}$ & $M$ & $S D$ & $M d n$ & $I Q R$ & $\mathrm{~N}$ & $M$ & $S D$ & $M d n$ & $I Q R$ & & \\
\hline Men & $82(66 \%)$ & & & & & $31(60 \%)$ & & & & & 0.56 & \\
\hline Age & 90 & 33.50 & 12.99 & 30.16 & 20.95 & 87 & 32.39 & 13.59 & 24.77 & 26.10 & 0.41 & \\
\hline Education & 90 & 13.90 & 2.76 & 13.00 & 4.00 & 87 & 13.97 & 2.44 & 13.00 & 4.00 & 0.65 & \\
\hline AST Congruency Cost & 90 & 110.52 & 87.96 & 97.12 & 132.01 & 85 & 80.77 & 73.13 & 76.08 & 98.45 & 0.02 & -0.20 \\
\hline AST Switch Cost & 90 & -102.28 & 95.03 & -107.99 & 125.61 & 85 & -106.50 & 91.34 & -99.97 & 113.94 & 0.94 & -0.01 \\
\hline AST \% Correct & 90 & 94.16 & 6.51 & 96.25 & 6.72 & 85 & 94.89 & 6.05 & 96.88 & 3.13 & 0.56 & -0.05 \\
\hline PAL Total Errors & 90 & 10.14 & 12.26 & 6.00 & 8.00 & 86 & 10.63 & 12.18 & 7.00 & 8.75 & 0.51 & 0.06 \\
\hline PRM Immediate & 90 & 92.13 & 9.54 & 91.67 & 16.67 & 85 & 92.25 & 9.34 & 91.67 & 16.67 & 0.99 & 0.00 \\
\hline PRM Delayed & 89 & 81.27 & 14.67 & 83.33 & 16.67 & 84 & 81.05 & 15.16 & 83.33 & 16.67 & 0.96 & 0.00 \\
\hline RVP A prime & 87 & 0.90 & 0.05 & 0.90 & 0.07 & 85 & 0.90 & 0.06 & 0.91 & 0.08 & 0.48 & -0.06 \\
\hline SRM \% Correct & 90 & 82.44 & 9.78 & 85.00 & 15.00 & 84 & 82.02 & 9.67 & 85.00 & 15.00 & 0.72 & 0.03 \\
\hline SSP Span Length & 88 & 6.74 & 1.47 & 7.00 & 3.00 & 84 & 6.73 & 1.44 & 7.00 & 3.00 & 0.95 & 0.01 \\
\hline SWM Between Errors & 90 & 15.92 & 16.58 & 11.00 & 22.75 & 85 & 14.78 & 16.28 & 8.00 & 19.00 & 0.76 & -0.03 \\
\hline SWM Strategy & 90 & 29.01 & 6.81 & 29.00 & 12.75 & 85 & 27.79 & 6.77 & 28.00 & 12.00 & 0.21 & -0.11 \\
\hline RTI Simple Reaction Time & 77 & 303.72 & 51.54 & 288.00 & 60.22 & 74 & 295.51 & 43.47 & 292.89 & 56.78 & 0.60 & -0.05 \\
\hline RTI 5-choice Reaction Time & 88 & 338.47 & 49.13 & 332.88 & 56.44 & 84 & 331.98 & 44.45 & 330.63 & 58.31 & 0.50 & -0.06 \\
\hline SOC Minimum Moves & 90 & 9.41 & 1.94 & 10.00 & 3.00 & 85 & 9.42 & 1.79 & 10.00 & 3.00 & 0.87 & 0.01 \\
\hline Matrix Reasoning & 84 & 28.01 & 4.70 & 28.00 & 6.00 & 85 & 27.95 & 4.30 & 29.00 & 5.00 & 0.95 & 0.01 \\
\hline Vocabulary & 83 & 57.72 & 8.54 & 59.00 & 12.00 & 83 & 56.93 & 8.57 & 57.00 & 11.50 & 0.59 & 0.05 \\
\hline TMT A & 89 & 26.62 & 8.33 & 25.00 & 10.00 & 86 & 26.22 & 8.64 & 25.00 & 10.50 & 0.70 & -0.03 \\
\hline TMT B & 88 & 65.82 & 25.77 & 60.00 & 35.25 & 86 & 64.37 & 24.55 & 58.50 & 22.75 & 0.75 & -0.03 \\
\hline Verbal fluency - Words & 88 & 38.36 & 13.64 & 38.00 & 16.00 & 84 & 37.92 & 10.01 & 36.50 & 14.25 & 0.90 & 0.01 \\
\hline Verbal fluency - Animals & 88 & 22.20 & 5.70 & 22.00 & 7.00 & 84 & 22.39 & 5.52 & 22.00 & 8.00 & 0.77 & -0.03 \\
\hline AVLT 1 to 5 & 86 & 49.31 & 10.14 & 51.50 & 14.00 & 83 & 51.18 & 7.95 & 52.00 & 11.00 & 0.35 & -0.08 \\
\hline AVLT Immediate & 86 & 10.57 & 2.87 & 11.00 & 4.00 & 83 & 11.04 & 2.54 & 11.00 & 4.00 & 0.36 & -0.08 \\
\hline AVLT Delayed & 85 & 10.39 & 3.06 & 11.00 & 4.00 & 82 & 10.94 & 2.67 & 11.00 & 4.00 & 0.27 & -0.10 \\
\hline Symbol Search & 87 & 33.52 & 6.70 & 33.00 & 9.00 & 86 & 34.06 & 6.65 & 33.00 & 7.00 & 0.81 & -0.02 \\
\hline Coding & 87 & 64.08 & 14.20 & 63.00 & 20.50 & 86 & 67.34 & 14.57 & 68.00 & 22.50 & 0.14 & -0.13 \\
\hline
\end{tabular}

${ }^{\mathrm{a}}$ Mann-Whitney U test; ${ }^{\mathrm{b}} \mathrm{a}$ Cliff's delta $(\Delta)$ of 0.11 is considered a small effect size, 0.28 is considered a medium effect size, and 0.43 is considered a large effect size.

Bold values indicate significance at $p<0.05$ without correction for multiple comparisons.

AST, Attention Switching Task; AVLT, Auditory Verbal Learning Test; IQR, interquartile range; LOC, loss of consciousness; M, mean; Mdn, median; PAL, Paired Associated Learning; PRM, Pattern Recognition Memory; RTI, Reaction Time; RVP, Rapid Visual Processing; SD, standard deviation; SOC, Stockings of Cambridge; SRM, Spatial Recognition Memory; SSP, Spatial Span; SWM, Spatial Working Memory; TMT, Trail Making Test.

( $p=0.043, \Delta=0.27$; Table 5). Regarding the remaining comparisons, no statistically significant differences were found, and the Cliff's delta effect sizes were small to very small.

There were no statistically significant differences in age, sex, or years of education between patients with $(n=87)$ and without witnessed LOC $(n=90)$. Compared with patients without witnessed LOC, patients with witnessed LOC differed less in reaction time between incongruent and congruent trials on the AST as indicated by the congruency cost ( $p=0.020, \Delta=-0.20$; Table 6$)$. Regarding the remaining comparisons between groups with and without LOC, no statistically significant differences were found, and the Cliff's delta effect sizes were small to very small.

There were no statistically significant differences in age, sex, or years of education between the patients with short $(<1 \mathrm{~h} ; n=125)$ and long (1-24h; $n=52)$ durations of PTA. Patients with $1-24 \mathrm{~h}$ of PTA performed worse than patients with $<1 \mathrm{~h}$ of PTA on 10 of the outcome measures: 3 from the CANTAB (RVP, $\Delta=0.25$; SWM-Strategy, $\Delta=0.21$; SOC, $\Delta=0.20$ ) and 7 from the paper-and-pencil battery (Matrix Reasoning, $\Delta=0.21$; Vocabulary, a test of verbal intelligence, considered to reflect pre-injury functioning, $\Delta=0.27$; Verbal fluency-phonemic trial, $\Delta=0.33$; Verbal fluency-semantic trial, $\Delta=0.26$; and all the three AVLT measures: trial $1-5, \Delta=0.25$; immediate recall, $\Delta=0.26$; and delayed recall, $\Delta=0.23$ (Table 7). The differences in terms of effect sizes (Cliff's delta) were small to medium for all tests, with the highest values for the verbal fluency trials.

\section{Discussion}

The present study is one of the largest studies of cognition in subacute mixed-mechanism MTBI, and the first to evaluate MTBIrelated differences in cognitive performance using the CENTERTBI battery, which is one of the largest ongoing studies in the history of brain injury research. We hypothesized group differences between the MTBI group and two control groups, TC participants and CC participants. We observed performances almost exclusively in the normal range, with comparable rates of impairment between patients with MTBIs and control participants. Subgroup analyses of patients with MTBI based on indices of injury severity revealed minimal differences based on the presence of LOC or intracranial traumatic lesions on CT or MRI. However, patients with longer durations of PTA performed more poorly on several CANTAB and paper-andpencil tests, a finding possibly confounded by lower estimated preinjury intellectual functioning as estimated by lower WASI Vocabulary performances in the group of patients with longer PTA.

Although patients with MTBI were evaluated only 2 weeks after injury, neuropsychological performances were similar between the MTBI and the control groups. According to some of the existing meta-analyses on neuropsychological outcomes after MTBI, group differences with greater effect sizes were expected. ${ }^{10,12,17,21}$ However, the view that subacute MTBI is associated with cognitive deficits is mostly based on results from studies conducted during 
Table 7. Comparing Patients Stratified by PTA

\begin{tabular}{|c|c|c|c|c|c|c|c|c|c|c|c|c|}
\hline & \multicolumn{5}{|c|}{$P T A<1$} & \multicolumn{5}{|c|}{ PTA $1-24 h$} & \multirow[b]{2}{*}{$\mathrm{P}^{\mathrm{a}}$} & \multirow[b]{2}{*}{$\Delta^{\mathrm{b}}$} \\
\hline & $\mathrm{N}$ & $M$ & $S D$ & $M d n$ & $I Q R$ & $\mathrm{~N}$ & $M$ & $S D$ & $M d n$ & $I Q R$ & & \\
\hline Men & $82(66 \%)$ & & & & & $31(60 \%)$ & & & & & 0.56 & \\
\hline Age & 125 & 33.37 & 13.42 & 29.35 & 24.30 & 52 & 31.96 & 12.95 & 25.47 & 18.27 & 0.70 & \\
\hline Education & 125 & 14.06 & 2.64 & 13.00 & 4.00 & 52 & 13.62 & 2.49 & 13.00 & 3.00 & 0.60 & \\
\hline AST Congruency Cost & 123 & 99.57 & 82.23 & 89.72 & 114.84 & 52 & 87.80 & 82.44 & 79.83 & 112.02 & 0.41 & -0.08 \\
\hline AST Switch Cost & 123 & -105.56 & 94.53 & -103.84 & 128.14 & 52 & -101.42 & 90.15 & -107.32 & 121.99 & 0.73 & 0.03 \\
\hline AST \% Correct & 123 & 94.40 & 6.58 & 96.88 & 5.94 & 52 & 94.78 & 5.59 & 96.25 & 5.00 & 0.68 & -0.04 \\
\hline PAL Total Errors & 124 & 9.81 & 11.56 & 6.00 & 10.00 & 52 & 11.75 & 13.61 & 7.00 & 8.25 & 0.29 & 0.10 \\
\hline PRM Immediate & 123 & 92.01 & 9.08 & 91.67 & 16.67 & 52 & 92.63 & 10.26 & 100.00 & 8.33 & 0.38 & -0.08 \\
\hline PRM Delayed & 122 & 82.10 & 13.58 & 83.33 & 16.67 & 51 & 78.92 & 17.51 & 83.33 & 25.00 & 0.47 & 0.07 \\
\hline RVP A prime & 121 & 0.91 & 0.05 & 0.91 & 0.06 & 51 & 0.89 & 0.05 & 0.89 & 0.08 & 0.01 & 0.25 \\
\hline SRM \% Correct & 123 & 82.80 & 9.71 & 85.00 & 12.50 & 51 & 80.88 & 9.63 & 80.00 & 15.00 & 0.15 & 0.14 \\
\hline SSP Span Length & 121 & 6.83 & 1.46 & 7.00 & 2.00 & 51 & 6.49 & 1.43 & 6.00 & 3.00 & 0.18 & 0.13 \\
\hline SWM Between Errors & 124 & 14.02 & 15.12 & 9.00 & 19.00 & 51 & 18.63 & 18.91 & 13.00 & 24.00 & 0.18 & 0.13 \\
\hline SWM Strategy & 124 & 27.68 & 6.72 & 28.00 & 12.00 & 51 & 30.22 & 6.72 & 31.00 & 10.50 & $\mathbf{0 . 0 3}$ & 0.21 \\
\hline RTI Simple Reaction Time & 108 & 299.33 & 49.70 & 293.58 & 61.11 & 43 & 300.63 & 43.09 & 288.00 & 47.86 & 0.80 & 0.03 \\
\hline RTI 5-choice Reaction Time & 122 & 335.09 & 49.76 & 327.33 & 61.31 & 50 & 335.81 & 39.42 & 335.19 & 39.10 & 0.60 & 0.05 \\
\hline SOC Minimum Moves & 124 & 9.58 & 1.90 & 10.00 & 3.00 & 51 & 9.02 & 1.73 & 9.00 & 2.00 & $\mathbf{0 . 0 3}$ & 0.20 \\
\hline Matrix Reasoning & 120 & 28.33 & 4.65 & 29.00 & 6.00 & 49 & 27.14 & 3.98 & 28.00 & 4.00 & 0.04 & 0.21 \\
\hline Vocabulary & 118 & 58.35 & 8.35 & 60.00 & 10.00 & 48 & 54.81 & 8.56 & 54.50 & 12.50 & 0.01 & 0.27 \\
\hline TMT A & 125 & 25.95 & 8.45 & 24.00 & 10.00 & 50 & 27.60 & 8.45 & 27.00 & 9.00 & 0.16 & 0.14 \\
\hline TMT B & 125 & 63.03 & 23.36 & 58.00 & 27.00 & 49 & 70.39 & 28.69 & 63.00 & 32.00 & 0.13 & 0.15 \\
\hline Verbal fluency - Words & 122 & 39.86 & 11.44 & 40.00 & 14.75 & 50 & 33.96 & 12.30 & 31.50 & 16.25 & $\mathbf{0 . 0 0}$ & 0.33 \\
\hline Verbal fluency - Animals & 122 & 22.95 & 5.41 & 23.00 & 6.00 & 50 & 20.70 & 5.78 & 19.50 & 7.75 & 0.01 & 0.26 \\
\hline AVLT 1 to 5 & 120 & 51.28 & 9.17 & 53.00 & 11.25 & 49 & 47.65 & 8.67 & 48.00 & 11.00 & 0.01 & 0.25 \\
\hline AVLT Immediate & 120 & 11.13 & 2.70 & 12.00 & 3.25 & 49 & 10.00 & 2.61 & 10.00 & 4.00 & 0.01 & 0.26 \\
\hline AVLT Delayed & 120 & 10.93 & 2.96 & 11.00 & 4.00 & 47 & 9.96 & 2.55 & 10.00 & 3.50 & 0.02 & 0.23 \\
\hline Symbol Search & 125 & 33.77 & 6.20 & 34.00 & 8.00 & 48 & 33.83 & 7.82 & 33.00 & 9.50 & 0.73 & 0.03 \\
\hline Coding & 125 & 66.46 & 14.14 & 66.00 & 21.00 & 48 & 63.71 & 15.15 & 60.50 & 22.00 & 0.23 & 0.12 \\
\hline
\end{tabular}

${ }^{\mathrm{a}}$ Mann-Whitney U test; ${ }^{\mathrm{b}}$ a Cliff's delta $(\Delta)$ of 0.11 is considered a small effect size, 0.28 is considered a medium effect size, and 0.43 is considered a large effect size.

Bold values indicate significance at $p<0.05$ without correction for multiple comparisons.

AST, Attention Switching Task; AVLT, Auditory Verbal Learning Test; IQR, interquartile range; M, mean; Mdn, median; PAL, Paired Associated Learning; PRM, Pattern Recognition Memory; PTA, post-traumatic amnesia; RTI, Reaction Time; RVP, Rapid Visual Processing; SD, standard deviation; SOC, Stockings of Cambridge; SRM, Spatial Recognition Memory; SSP, Spatial Span; SWM, Spatial Working Memory; TMT, Trail Making Test.

the first few days after injury. Based on our findings, substantial cognitive recovery most likely occurs before 2 weeks after injury. There are few studies of cognition at 2 weeks after MTBI, and those identified have produced similar results. ${ }^{22-25}$

Despite the general view that cognitive deficits in patients with MTBI tend to resolve rapidly after injury, subgroups of patients with MTBI may suffer from trauma-related cognitive impairments that might not be revealed by meta-analyses. ${ }^{20,53}$ The current study evaluated three subgroups based on indices of injury severity: LOC, PTA, and positive neuroimaging. First, we compared patients with visible traumatic lesions on brain CT or MRI (i.e., complicated MTBI) with those without such lesions (i.e., uncomplicated MTBI). This comparison revealed only one difference on the Verbal fluency test (phonemic trial). Among the CENTER-TBI battery tests, none of the paper-and-pencil or CANTAB tests differentiated between patients with complicated and uncomplicated MTBI. This negligible difference between complicated and uncomplicated MTBI was in line with several other studies. ${ }^{54-59}$ Some studies have reported that the subacute effects of MTBI on cognition are more pronounced in patients with complicated MTBI, ${ }^{2,60}$ but all patients in the latter studies were recruited from the ED at a trauma hospital, and are therefore less representative of patients with MTBI than the patients in our study (i.e., both an ED and outpatient sample).

Similar to our results on neuroimaging findings, there was only one significant difference between patients with LOC and patients without LOC on cognitive outcomes. Patients with LOC were faster on incongruent trials on the AST. This group difference is in a non-intuitive direction and is likely an incidental finding among the many non-significant group differences based on LOC.

Several previous studies on sports-related concussion have revealed worse cognitive outcome in patients with LOC than patients without LOC. ${ }^{16}$ In contrast, a study on patients from a tertiary trauma center found no difference between patients with LOC, no LOC, or uncertain LOC. ${ }^{61}$ None of these studies, however, tested patients beyond 1 week post-injury. Moreover, another study found no effect of LOC on cognitive functioning even within the first $24 \mathrm{~h}$ after MTBI. ${ }^{62}$ One reason for inconsistent results on cognitive functioning and LOC may be attributable to poor measurement of LOC, which is often based on self-report or informant-report and may not be properly recorded by physicians in emergency settings. Further, patients without an eyewitness may struggle to differentiate between LOC and PTA, and unlike many studies, we recorded LOC only when witnessed. Therefore, in the present study, we cannot rule out that patients coded as not having LOC may have been, at least briefly, unconscious. A less conservative approach in defining LOC, might have resulted in even smaller differences between the groups, so we consider our results to justify that LOC may not serve as an important variable in describing injury severity, at least in this cohort. 
In contrast to the findings on neuroimaging and LOC, duration of PTA emerged as a possible marker of subacute cognitive impairments. Patients with PTA $>1 \mathrm{~h}$ had significantly worse neuropsychological performance than patients with shorter durations of PTA. The differences were not large, but were found across several tests of executive function, verbal memory, and processing speed. In patients with longer PTA, differences were also evident on tests included in the CENTER-TBI battery. The association between the duration of PTA and neuropsychological outcome aligns with a smaller study examining memory ${ }^{63}$ and another study where patients were tested in the ED. ${ }^{64}$ In sport-related concussion research, PTA has been identified as an injury characteristic predictive of reduced neuropsychological functioning after 10 days post-injury. ${ }^{30,31}$ An unexpected finding, however, was that the WASI Vocabulary scores and Matrix Reasoning scores, two measures of intellectual functioning, were lower in the group with longer PTA. Verbal intelligence, in particular, is regarded to be unaffected by $\mathrm{MTBI}^{65}$ and is often used as a proxy for pre-injury functioning. ${ }^{66}$

This finding could indicate an imbalance in intellectual functioning between the PTA groups. We therefore re-ran the analyses where the differences between the PTA groups were significant with a nonparametric analysis of covariance (ANCOVA) ${ }^{67}$ and included vocabulary scores and age as covariates. The only difference that remained significant between the PTA groups was on SWM-Strategy, a CANTAB test of working memory $(p=0.042)$. We therefore consider it likely that the observed differences in cognition between patients with long and short PTA were mostly explained by differences in pre-morbid intelligence in this cohort. To the best of our knowledge, our study is the first to compare performances on the CANTAB between participants with and without MTBI. Moreover, it is the first to assess participants with and without MTBI using the CANTAB alongside paper-and-pencil neuropsychological tests. In our study, only the subgroup analyses comparing cognitive function based on PTA duration revealed consistent differences. These differences were observed both on tests from the CANTAB and on paper-and-pencil tests, with mostly small effect sizes for both types of tests. Based on these findings, the CANTAB seems to be neither more, nor less, suitable in detecting subtle cognitive impairments than the paper-and-pencil tests.

This study includes a collection of strengths and weaknesses that both enhance and reduce the implications of the findings. Notably, this is one of few population-based studies of patients with MTBI that include patients evaluated in primary care settings and a hospital trauma center. The study involved many participants at the mildest end of the spectrum of MTBI severity. That makes the findings less generalizable to cohorts with more severe injuries (i.e., a GCS score of 13-14, and greater percentages with complicated MTBIs) who may present to Level 1 trauma centers, but more generalizable to the larger population of patients with MTBI who experience milder injuries and present to outpatient clinics or do not seek care. However, in studies like ours, many patients will have normal neuroimaging, and the majority will have a GCS score of 15 and a short duration of PTA, which reduces the statistical power to analyze the effects of such indices of injury severity. It was also a strength of our study that two different control groups were recruited, comparing post-MTBI performances with performances following orthopedic trauma and no trauma at all.

That the presentation of effect sizes applied to both the CANTAB and paper-and-pencil tests is also a strength of the study. Despite the large number of meta-analyses in the field, this study adds new knowledge by illustrating that MTBI is not related to measurable cognitive impairments at 2 weeks post-injury. Relatively few studies have examined neuropsychological outcome at this time-point. An important methodological issue is that we conducted numerous exploratory statistical tests with no corrections for multiple comparisons. This was by design. The small number of significant findings, without question, could be spurious. The focus of the study was, in fact, exploratory and we wanted to compare effect sizes across 25 individual neuropsychological test scores, some computerized and some traditional, and most, but not all, in the CENTER-TBI battery.

\section{Conclusion}

There are several noteworthy findings from this study. First, this large sample of adults with MTBIs did not show evidence of cognitive deficits at approximately 2 weeks following injury. Second, those who experienced LOC at the time of injury did not perform more poorly on neuropsychological testing than those who did not. Third, those with complicated MTBIs performed comparably on testing compared with those with uncomplicated MTBIs. Fourth, those with greater duration of PTA did not perform worse on neuropsychological testing after controlling for pre-injury differences in intellectual functioning. Finally, computerized tests included in the CENTER-TBI battery were no more, or less, sensitive to the subtle cognitive effects of MTBI than traditional paperand-pencil neuropsychological tests.

\section{Acknowledgments}

The authors are grateful to the patients and community controls, the staff of the Trondheim Municipal Emergency Department, the Department of Neurosurgery, the Department of Anesthesiology and Insensitive Care Medicine, and the Department of Radiology and Nuclear Medicine at St. Olav's Hospital, Trondheim University Hospital, and to Ingeborg Nakken, Jian Xu, Kent Gøran Moen, and Kjell Arne Kvistad for their cooperation with the MRI examinations, and to Stine Bjøralt for study coordination.

\section{Funding Information}

This work was supported by the Liaison Committee between the Central Norway Regional Health Authority and the Norwegian University of Science and Technology.

\section{Author Disclosure Statement}

Dr. Grant Iverson has received research support from test publishing companies in the past, including ImPACT ${ }^{\circledR}$ Applications Systems, Psychological Assessment Resources, Inc. (PAR, Inc.), and CNS Vital Signs. He receives royalties from PAR, Inc. for the WCST-64. He has a clinical practice in forensic neuropsychology, including expert testimony, involving individuals who have sustained MTBIs. He has received grant funding from the National Football League and salary support from the Harvard Integrated Program to Protect and Improve the Health of NFLPA Members. He serves as a scientific advisor for BioDirection, Inc., SWAY Operations, LLC, and Highmark, Inc. He acknowledges unrestricted philanthropic support from the Mooney-Reed Charitable Foundation, ImPACT ${ }^{\circledR}$ Applications, Inc., the Heinz Family Foundation, and the Spaulding Research Institute. The remaining authors have no competing financial interests to disclose.

\section{References}

1. McCrory, P., Meeuwisse, W.H., Echemendia, R.J., Iverson, G.L., Dvorak, J., and Kutcher, J.S. (2013). What is the lowest threshold to make a diagnosis of concussion? Br. J. Sports Med. 47, 268-271. 
2. Iverson, G.L. (2006). Complicated vs. uncomplicated mild traumatic brain injury: acute neuropsychological outcome. Brain Inj. 20, 13351344.

3. Kashluba, S., Hanks, R.A., Casey, J.E., and Millis, S.R. (2008). Neuropsychologic and functional outcome after complicated mild traumatic brain injury. Arch. Phys. Med. Rehabil. 89, 904-911.

4. Skandsen, T., Einarsen, C.E., Normann, I., Bjoralt, S., Karlsen, R.H., McDonagh, D., Nilsen, T.L., Akslen, A.N., Haberg, A.K., and Vik, A. (2018). The epidemiology of mild traumatic brain injury: the Trondheim MTBI follow-up study. Scand. J. Trauma Resusc. Emerg. Med. 26,34 .

5. Barkhoudarian, G., Hovda, D.A., and Giza, C.C. (2016). The Molecular Pathophysiology of Concussive Brain Injury-an update. Phys. Med. Rehabil. Clin. N. Am. 27, 373-393.

6. Carroll, L.J., Cassidy, J.D., Peloso, P.M., Borg, J., von Holst, H., Holm, L., Paniak, C., and Pepin, M. (2004). Prognosis for mild traumatic brain injury: results of the WHO Collaborating Centre Task Force on Mild Traumatic Brain Injury. J. Rehabil. Med. (43 Suppl.), $84-105$

7. Williams, R.M., Puetz, T.W., Giza, C.C., and Broglio, S.P. (2015). Concussion recovery time among high school and collegiate athletes: a systematic review and meta-analysis. Sports Med. (Auckland, N.Z.) $45,893-903$

8. Carroll, L.J., Cassidy, J.D., Cancelliere, C., Cote, P., Hincapie, C.A., Kristman, V.L., Holm, L.W., Borg, J., Nygren-de Boussard, C., and Hartvigsen, J. (2014). Systematic review of the prognosis after mild traumatic brain injury in adults: cognitive, psychiatric, and mortality outcomes: results of the International Collaboration on Mild Traumatic Brain Injury Prognosis. Arch. Phys. Med. Rehabil. 95, S152S173.

9. Broglio, S.P., and Puetz, T.W. (2008). The effect of sport concussion on neurocognitive function, self-report symptoms and postural control : a meta-analysis. Sports Med. 38, 53-67.

10. Rohling, M.L., Binder, L.M., Demakis, G.J., Larrabee, G.J., Ploetz, D.M., and Langhinrichsen-Rohling, J. (2011). A meta-analysis of neuropsychological outcome after mild traumatic brain injury: reanalyses and reconsiderations of Binder et al. (1997), Frencham et al (2005), and Pertab et al. (2009). Clin. Neuropsychol. 25, 608-623.

11. Belanger, H.G., Spiegel, E., and Vanderploeg, R.D. (2010). Neuropsychological performance following a history of multiple selfreported concussions: a meta-analysis. J. Int. Neuropsychol. Soc.16, 262-267.

12. Belanger, H.G., Curtiss, G., Demery, J.A., Lebowitz, B.K., and Vanderploeg, R.D. (2005). Factors moderating neuropsychological outcomes following mild traumatic brain injury: a meta-analysis. J. Int Neuropsychol. Soc. 11, 215-227.

13. Belanger, H.G., and Vanderploeg, R.D. (2005). The neuropsychological impact of sports-related concussion: a meta-analysis. J. Int Neuropsychol. Soc.11, 345-357.

14. Binder, L.M., Rohling, M.L., and Larrabee, G.J. (1997). A review of mild head trauma. Part I: meta-analytic review of neuropsychological studies. J. Clin. Exp. Neuropsychol. 19, 421-431.

15. Dougan, B.K., Horswill, M.S., and Geffen, G.M. (2014). Athletes' age, sex, and years of education moderate the acute neuropsychological impact of sports-related concussion: a meta-analysis. J. Int. Neuropsychol. Soc. 20, 64-80.

16. Dougan, B.K., Horswill, M.S., and Geffen, G.M. (2014). Do injury characteristics predict the severity of acute neuropsychological deficits following sports-related concussion? A meta-analysis. J. Int. Neuropsychol. Soc. 20, 81-87.

17. Frencham, K.A., Fox, A.M., and Maybery, M.T. (2005). Neuropsychological studies of mild traumatic brain injury: a meta-analytic review of research since 1995. J. Clin. Exp. Neuropsychol. 27, 334351

18. Karr, J.E., Areshenkoff, C.N., Duggan, E.C., and Garcia-Barrera, M.A. (2014). Blast-related mild traumatic brain injury: a Bayesian random-effects meta-analysis on the cognitive outcomes of concussion among military personnel. Neuropsychol. Rev. 24, 428-444.

19. Karr, J.E., Areshenkoff, C.N., and Garcia-Barrera, M.A. (2014). The neuropsychological outcomes of concussion: a systematic review of meta-analyses on the cognitive sequelae of mild traumatic brain injury. Neuropsychology 28, 321-336.

20. Pertab, J.L., James, K.M., and Bigler, E.D. (2009). Limitations of mild traumatic brain injury meta-analyses. Brain Inj. 23, 498-508.
21. Schretlen, D.J., and Shapiro, A.M. (2003). A quantitative review of the effects of traumatic brain injury on cognitive functioning Int. Rev. Psychiatry 15, 341-349.

22. Mayer, A.R., Hanlon, F.M., Dodd, A.B., Ling, J.M., Klimaj, S.D., and Meier, T.B. (2015). A functional magnetic resonance imaging study of cognitive control and neurosensory deficits in mild traumatic brain injury. Hum. Brain Mapp. 36, 4394-4406.

23. Waljas, M., Lange, R.T., Hakulinen, U., Huhtala, H., Dastidar, P., Hartikainen, K., Ohman, J., and Iverson, G.L. (2014). Biopsychosocial outcome after uncomplicated mild traumatic brain injury. J. Neurotrauma 31, 108-124.

24. Mayer, A.R., Ling, J., Mannell, M.V., Gasparovic, C., Phillips, J.P., Doezema, D., Reichard, R., and Yeo, R.A. (2010). A prospective diffusion tensor imaging study in mild traumatic brain injury. Neurology 74, 643-650.

25. Nelson, L.D., Furger, R.E., Gikas, P., Lerner, E.B., Barr, W.B., Hammeke, T.A., Randolph, C., Guskiewicz, K., and McCrea, M.A (2017). Prospective, head-to-head study of three computerized neurocognitive assessment tools Part 2: utility for assessment of mild traumatic brain injury in emergency department patients. J. Int. Neuropsychol. Soc. 23, 293-303.

26. Burton, A. (2017). The CENTER-TBI core study: the making-of. Lancet. Neurol. 16, 958-959.

27. Wheble, J.L., and Menon, D.K. (2016). TBI-the most complex disease in the most complex organ: the CENTER-TBI trial-a commentary. J. R. Army Med. Corps 162, 87-89.

28. Maas, A.I., Menon, D.K., Steyerberg, E.W., Citerio, G., Lecky, F. Manley, G.T., Hill, S., Legrand, V., Sorgner, A., and CENTER-TBI Participants and Investigators. (2015). Collaborative European NeuroTrauma Effectiveness Research in Traumatic Brain Injury (CENTER-TBI): a prospective longitudinal observational study. Neurosurgery 76, 67-80.

29. Fowler, K.S., Saling, M.M., Conway, E.L., Semple, J.M., and Louis, W.J. (2002). Paired associate performance in the early detection of DAT. J. Int. Neuropsychol. Soc. 8, 58-71.

30. Juncos-Rabadan, O., Pereiro, A.X., Facal, D., Reboredo, A. and LojoSeoane, C. (2014). Do the Cambridge Neuropsychological Test Automated Battery episodic memory measures discriminate amnestic mild cognitive impairment? Int. J. Geriatr. Psychiatry 29, 602-609.

31. Lees, J., Michalopoulou, P.G., Lewis, S.W., Preston, S., Bamford, C., Collier, T., Kalpakidou, A., Wykes, T., Emsley, R., Pandina, G., Kapur, S., and Drake, R.J. (2017). Modafinil and cognitive enhancement in schizophrenia and healthy volunteers: the effects of test battery in a randomised controlled trial. Psychol. Med. 47, 2358-2368.

32. Pearce, A.J., Hoy, K., Rogers, M.A., Corp, D.T., Davies, C.B., Maller, J.J., and Fitzgerald, P.B. (2015). Acute motor, neurocognitive and neurophysiological change following concussion injury in Australian amateur football. A prospective multimodal investigation. J. Sci. Med. Sport 18, 500-506.

33. Pearce, A.J., Hoy, K., Rogers, M.A., Corp, D.T., Maller, J.J., Drury, H.G., and Fitzgerald, P.B. (2014). The long-term effects of sports concussion on retired Australian football players: a study using transcranial magnetic stimulation. J. Neurotrauma 31, 1139-1145.

34. Pearce, A.J., Rist, B., Fraser, C.L., Cohen, A., and Maller, J.J. (2018) Neurophysiological and cognitive impairment following repeated sports concussion injuries in retired professional rugby league players. Brain Inj. 32, 498-505.

35. Sterr, A., Herron, K.A., Hayward, C., and Montaldi, D. (2006). Are mild head injuries as mild as we think? Neurobehavioral concomitants of chronic post-concussion syndrome. BMC Neurol. 6, 7.

36. Barnett, J.H., Blackwell, A.D., Sahakian, B.J., and Robbins, T.W. (2016). The Paired Associates Learning (PAL) Test: 30 Years of CANTAB translational neuroscience from laboratory to bedside in dementia research. Curr. Top. Behav. Neurosci. 28, 449-474.

37. Stenberg, J., Haberg, A.K., Follestad, T., Olsen, A., Iverson, G.L., Terry, D.P., Karlsen, R.H., Saksvik, S.B., Karaliute, M., Ek, J.A.N Skandsen, T., and Vik, A. (2020). Cognitive reserve moderates cognitive outcome after mild traumatic brain injury. Arch. Phys. Med. Rehabil. 101, 72-80.

38. Chaban, V., Clarke, G., Skandsen, T., Islam, R., Einarsen, C., Vik, A., Damas, J.K., Mollnes, T.E., Haberg, A.K., and Pischke, S.E. (2020). Systemic inflammation persists the first year after mild traumatic brain injury: results from the prospective Trondheim Mild TBI Study. J. Neurotrauma 37, 2120-2130. 
39. Einarsen, C.E., Moen, K.G., Haberg, A.K., Eikenes, L., Kvistad, K.A., Xu, J., Moe, H.K., Tollefsen, M.H., Vik, A., and Skandsen, T. (2019). Patients with Mild Traumatic Brain Injury Recruited from Both Hospital and Primary Care Settings: A Controlled Longitudinal Magnetic Resonance Imaging Study. J. Neurotrauma 36, 3172-3182.

40. Carroll, L.J., Cassidy, J.D., Holm, L., Kraus, J., and Coronado, V.G. (2004). Methodological issues and research recommendations for mild traumatic brain injury: the WHO Collaborating Centre Task Force on Mild Traumatic Brain Injury. J. Rehabil. Med. 36, 113-125.

41. Strauss, E., Sherman, E.M.S., and Spreen, O. (2006 ). A Compendium of Neuropsychological Tests. Administration, Norms, and Commentary, 3rd ed. Oxford University Press: New York.

42. Lezak, M.D., Howieson, D.B., Bigler, E.D., and Tranel, D. (2012). Neuropsychological Assessment, 5th revised ed. Oxford University Press Inc: New York.

43. Mitrushina, M.N., Boone, K.B., Razani, J., and D'Elia, L.F. (2005). Handbook of Normative Data for Neuropsychological Assessment, 2nd ed. Oxford University Press: Oxford.

44. Weschler, D. (2011). Wechsler Adult Intelligence Scale (Norwegian version), 4th ed. Pearson Assessment: San Antonio, TX

45. Weschler, D. (2008). Wechsler Adult Intelligence Scale, 4th ed. Pearson Assessment: San Antonio, TX.

46. Schmidt, M. (1996). Rey Auditory-Verbal Learning Test. Western Psychological Services: Los Angeles, CA.

47. Tombaugh, T.N., Kozak, J., and Rees, L. (1999). Normative data stratified by age and education for two measures of verbal fluency: FAS and animal naming. Arch. Clin. Neuropsychol. 14, 167-177.

48. Weschler, D. (2007). Wechsler Abbreviated Scale of Intelligence (Norwegian version). Pearson Assessment: San Antonio, TX.

49. Weschler, D. (1999). Wechlser Abbreviated Scale of Intelligence. The Psychological Corporation: San Antonio, TX.

50. Kampfl, A., Franz, G., Aichner, F., Pfausler, B., Haring, H.P., Felber, S., Luz, G., Schocke, M., and Schmutzhard, E. (1998). The persistent vegetative state after closed head injury: clinical and magnetic resonance imaging findings in 42 patients. J. Neurosurg. 88, 809-816.

51. Wilson, J.T., Wiedmann, K.D., Hadley, D.M., Condon, B., Teasdale, G., and Brooks, D.N. (1988). Early and late magnetic resonance imaging and neuropsychological outcome after head injury. J. Neurol. Neurosurg. Psychiatry 51, 391-396.

52. Vargha, A., and Delaney, H.D. (2000). A critique and improvement of the CL common language effect size statistics of McGraw and Wong. J. Ed. Behav. Stat. 25, 101-132.

53. Iverson, G.L. (2010). Mild traumatic brain injury meta-analyses can obscure individual differences. Brain Inj. 24, 1246-1255.

54. de Guise, E., Lepage, J.F., Tinawi, S., LeBlanc, J., Dagher, J., Lamoureux, J., and Feyz, M. (2010). Comprehensive clinical picture of patients with complicated vs. uncomplicated mild traumatic brain injury. Clin. Neuropsychol. 24, 1113-1130.

55. Sadowski-Cron, C., Schneider, J., Senn, P., Radanov, B.P., Ballinari, P., and Zimmermann, H. (2006). Patients with mild traumatic brain injury: immediate and long-term outcome compared to intra-cranial injuries on CT scan. Brain Inj. 20, 1131-1137.

56. Iverson, G.L., Lange, R.T., Waljas, M., Liimatainen, S., Dastidar, P., Hartikainen, K.M., Soimakallio, S., and Ohman, J. (2012). Outcome from complicated versus uncomplicated mild traumatic brain injury. Rehabil. Research Pract. 2012, 415740.

57. Hughes, D.G., Jackson, A., Mason, D.L., Berry, E., Hollis, S., and Yates, D.W. (2004). Abnormalities on magnetic resonance imaging seen acutely following mild traumatic brain injury: correlation with neuropsychological tests and delayed recovery. Neuroradiology 46, 550-558.

58. Lange, R.T., Iverson, G.L., and Franzen, M.D. (2009). Neuropsychological functioning following complicated vs. uncomplicated mild traumatic brain injury. Brain Inj. 23, 83-91.

59. Lee, H., Wintermark, M., Gean, A.D., Ghajar, J., Manley, G.T., and Mukherjee, P. (2008). Focal lesions in acute mild traumatic brain injury and neurocognitive outcome: CT versus 3 T MRI. J. Neurotrauma 25, 1049-1056.

60. Dikmen, S., Machamer, J., and Temkin, N. (2017). Mild traumatic brain injury: longitudinal study of cognition, functional status, and post-traumatic symptoms. J. Neurotrauma 34, 1524-1530.

61. Iverson, G.L., Lovell, M.R., and Smith, S.S. (2000). Does brief loss of consciousness affect cognitive functioning after mild head injury? Arch. Clin. Neuropsychol. 15, 643-648.

62. de Freitas Cardoso, M.G., Faleiro, R.M., de Paula, J.J., Kummer, A., Caramelli, P., Teixeira, A.L., de Souza, L.C., and Miranda, A.S. (2019). Cognitive impairment following acute mild traumatic brain injury. Front. Neurol. 10, 198.

63. Stulemeijer, M., Vos, P.E., van der Werf, S., van Dijk, G., Rijpkema, M., and Fernandez, G. (2010). How mild traumatic brain injury may affect declarative memory performance in the post-acute stage. J. Neurotrauma 27, 1585-1595.

64. Shores, E.A., Lammel, A., Hullick, C., Sheedy, J., Flynn, M., Levick, W., and Batchelor, J. (2008). The diagnostic accuracy of the Revised Westmead PTA Scale as an adjunct to the Glasgow Coma Scale in the early identification of cognitive impairment in patients with mild traumatic brain injury. J. Neurol. Neurosurg. Psychiatry 79, 1100-1106.

65. Fisher, D.C., Ledbetter, M.F., Cohen, N.J., Marmor, D., and Tulsky, D.S. (2000). WAIS-III and WMS-III profiles of mildly to severely brain-injured patients. Appl. Neuropsychol. 7, 126-132.

66. Levi, Y., Rassovsky, Y., Agranov, E., Sela-Kaufman, M., and Vakil, E. (2013). Cognitive reserve components as expressed in traumatic brain injury. J. Int. Neuropsychol. Soc. 19, 664-671.

67. Quade, D. (1967). Rank analysis of covariance. J. Am. Stat. Assoc. 62, 1187-1200.

Address correspondence to:

Toril Skandsen, $M D, P h D$

Department of Neuromedicine and Movement Science Faculty of Medicine and Health Sciences

Norwegian University of Science and Technology, NTNU

Trondheim N-7491

Norway

E-mail: toril.skandsen@ntnu.no 